\title{
Ore-Waste Discrimination in Epithermal Deposits Using Near-Infrared to Short-Wavelength Infrared (NIR-SWIR) Hyperspectral Imagery
}

\author{
M. Dalm ${ }^{1}$ (D) M. W. N. Buxton ${ }^{1}$. \\ F. J. A. van Ruitenbeek ${ }^{2}$
}

Received: 29 September 2017 / Accepted: 24 June 2018 / Published online: 16 July 2018

(C) The Author(s) 2018

\begin{abstract}
Near-infrared (NIR) and short-wavelength infrared (SWIR) hyperspectral imagery can be used to detect certain alteration minerals. At epithermal deposits, the formation of alteration minerals is, in theory, related to the mineralisation of gold and silver. In order to provide foundations for developing sensor-based sorting applications at a mine that exploits such a deposit, it was investigated if NIR-SWIR hyperspectral imagery can be used to distinguish between ore and waste particles by characterising the alteration mineralogy. Maps were produced from the NIR-SWIR hyperspectral images of 827 drill core samples that show mineral occurrences, mineral absorption feature intensities and characteristics of the iron oxide mineralogy. Partial least squares discriminant analysis (PLS-DA) was applied to the information contained in these maps to investigate if this information can be used to discriminate between ore and waste. The results showed that NIR-SWIR hyperspectral imagery could be used to segment a population of waste samples by detecting occurrences of pyrophyllite, dickite and/or illite. This result can be explained by the fact that these minerals are commonly deposited further away from the ore-bearing epithermal veins, while the absence of SWIR-active minerals or detected occurrences of alunite are more closely associated with these structures. The ability to identify waste with NIR-SWIR spectral sensors means there is potential that sensor-based sorting can be used to remove this waste from mineral processing operations. Additional research is still required to assess the economic feasibility of such a sensor-based sorting application.
\end{abstract}

$\triangle \quad$ M. Dalm

M.Dalm@TUDelft.nl

1 Resource Engineering, Faculty of Civil Engineering and Geosciences, Delft University of Technology, P.O. Box 5048, 2600 GA Delft, The Netherlands

2 Department of Earth Systems Analysis, Faculty of Geo-Information Science and Earth Observation (ITC), University of Twente, P.O. Box 6, 7500 AA Enschede, The Netherlands 
Keywords Sensor-based sorting · Ore sorting $\cdot$ Epithermal Au $\cdot$ Near-infrared $\cdot$ NIR . Short-wavelength infrared $\cdot$ SWIR $\cdot$ Hyperspectral imaging

\section{Introduction}

The ability to identify sub-economic ore with near-infrared (NIR) and/or shortwavelength infrared (SWIR) spectral sensors allows sensor-based sorting applications to be developed that can be used to remove this material from the feed of mineral processing operations. Sensor-based sorting is the mechanical separation of singular particles on certain physical and/or chemical properties after determining these properties with a sensor. Since this technique is generally cheaper than the conventional mineral processing methods, pre-concentrating mined ore with sensor-based sorting can potentially reduce mineral processing costs (Buxton and Benndorf 2013; Lessard et al. 2014; Salter and Wyatt 1991; Sivamohan and Forssberg 1991; Wills and Finch 2016).

Hyperspectral imagery data are often represented as a cube containing spatial information along the first two dimensions and spectral information along the third. The spectral information that is commonly recorded with NIR-SWIR hyperspectral imagers is the diffuse reflectance on wavelengths ranging from 450 to $2500 \mathrm{~nm}$. Within this spectral range, certain minerals absorb radiation on specific wavelength bands. These minerals can be identified by analysing the wavelength positions, shapes and relative intensities of the absorption features they produce. Absorption features on the NIR range (450-1300 $\mathrm{nm}$ ) are produced by electronic molecular processes, such as charge transfer, crystal field effects, conduction band transitions and colour centres (Clark 1999; Hunt 1977). On the SWIR range (1300-2500 nm), absorption features are produced by changes in the vibrational energy levels of molecule bonds (Clark 1999; Hollas 2004). It depends on the composition and structure of the mineral whether any of these processes can occur and not all minerals can, therefore, be detected with NIRSWIR spectral sensors. Minerals that can be detected are referred to as NIR-active or SWIR-active minerals.

Recent studies by Dalm et al. $(2014,2017)$ showed that NIR-SWIR spectral sensors can be used to segment sub-economic particles from ore samples obtained from a porphyry copper deposit. This was based on detecting the occurrence of specific alteration minerals of which the formation is related to the mineralisation of copper. The mineralisation of gold and silver at epithermal deposits is, in theory, also related to certain hydrothermal alteration zones (Pirajno 1992; Sillitoe 1993; Simmons et al. 2005). It was, therefore, investigated if NIR-SWIR hyperspectral imagery can be used to discriminate between ore and waste for a collection of 827 drill core samples that were obtained from a high-sulphidation epithermal gold-silver deposit.

The aim of the study presented in this paper was to use NIR-SWIR hyperspectral imagery to characterise the alteration mineralogy of samples in order to use the mineralogical data to segment a population of samples with gold grades $<0.20 \mathrm{ppm}$. This is an assumed cut-off grade that is used to distinguish between ore and waste, which is realistic for economic processing of the ore on which this study is based. This paper also introduces a novel method in which partial least squares discriminant analysis (PLS-DA) is used to produce mineral occurrence maps from the measured NIR-SWIR 
hyperspectral images in order to extract mineralogical information. PLS-DA is also applied to investigate if this mineralogical information can be used to distinguish between ore and waste.

\section{Hydrothermal Alteration at Epithermal Gold-Silver Deposits}

Epithermal gold-silver deposits form at depths up to $1500 \mathrm{~m}$ below the surface, where sharp pressure and temperature gradients result in boiling of the hydrothermal fluids, which changes fluid composition and forces gold and silver to precipitate (Pirajno 1992; Simmons et al. 2005). Two different types of epithermal deposits are generally distinguished. The first is a high-sulphidation type, which is formed by acidic, sulphurrich and oxidised hydrothermal fluids. The second is a low-sulphidation type, which is formed by near-neutral, sulphur-poor and reduced hydrothermal fluids (Sillitoe 1993; Simmons et al. 2005).

The study described in this paper is based on samples obtained from a highsulphidation epithermal deposit. Figure 1 presents a schematic model of the alteration zoning that generally occurs near epithermal veins and breccias of this deposit type. It can be observed from this figure that the main ore zone is associated with the vuggy quartz alteration. However, the alteration-mineralisation relationship can be affected by later alteration events that overprint the previously formed alteration mineralogy (Pirajno 1992). The nature of these relationships can, therefore, vary between individual deposits.

\section{PLS-DA}

PLS-DA is a statistical method in which a partial least squares (PLS) regression is used to investigate the possibilities of classifying samples into certain categories by using a set of predictor variables. PLS regression is a method that is used to fit a multivariate linear regression model between the predictor variables and one or more response variables (Næs and Martens 1984; Wold et al. 2001). The difference between PLS-DA and a standard PLS regression is that, during model calibration, the response variables are replaced by a dummy matrix with assigned class memberships (Sjöström et al. 1986).

PLS regression is based on a projection of the original data on a set of orthogonal components that describe the maximum covariance between the predictor data and the responses (Abdi 2010; Wold et al. 2001). These orthogonal components are commonly referred to as latent variables. The advantage of PLS regression over most other methods for calibrating multivariate linear regression models is that, by using the latent variables, the model is able to handle strongly collinear and noisy predictor data (Wold et al. 2001). Furthermore, the PLS regression model uses so-called scores and loadings for the samples and predictor variables, respectively, that allow the predictor variables to be identified that are most relevant for producing certain classifications.

When PLS-DA is applied, it is important to select the optimal number of latent variables that are used by the model. A higher number of latent variables usually increases the accuracy of the model on data used for calibration, but the predictive power on new data may be decreased due to overfitting (Wold et al. 2001; Abdi 2010). 


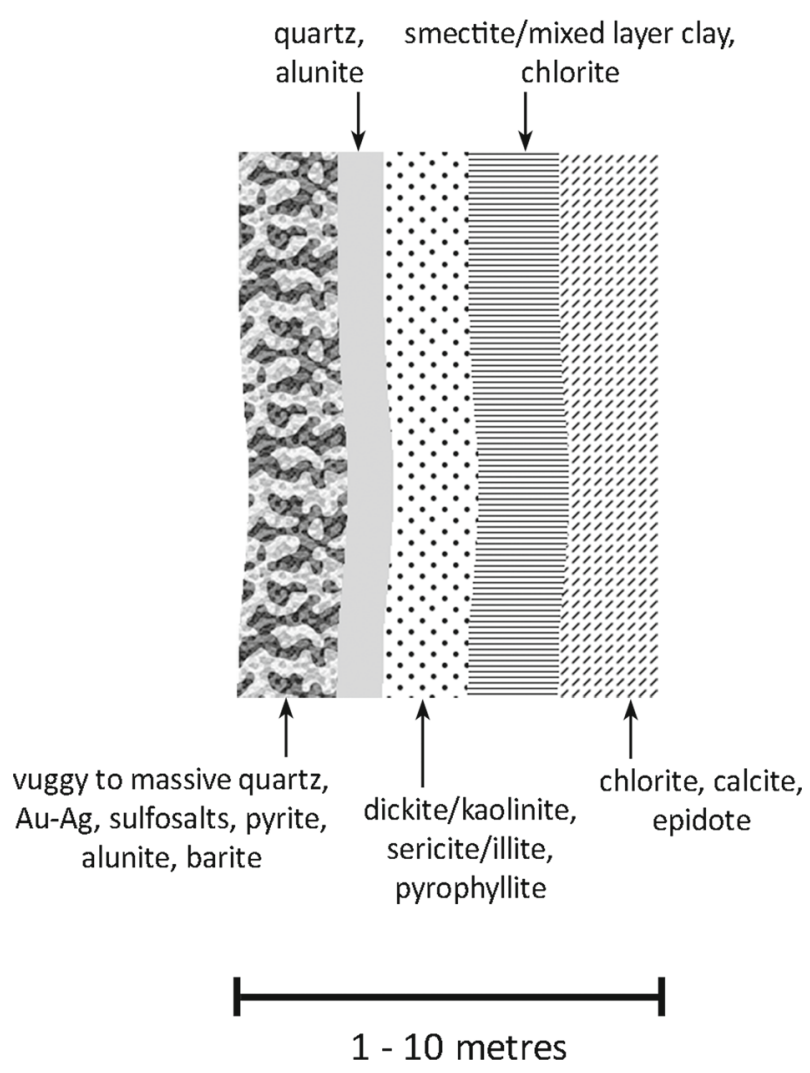

Fig. 1 Schematic alteration zoning outward (left to right) from high-sulphidation epithermal alteration veins or other geometrical forms [after Sillitoe (1993) and Simmons et al. (2005)]. The patterns result from fluid-rock interactions and declining fluid temperatures

A common method for selecting the optimal number of latent variables is by removing a fraction of the samples from the dataset during model calibration. The fraction that is removed is usually around $20-35 \%$ and is referred to as the validation set or test set. The error rate of classification on the calibration and validation subsets are compared when the model is calibrated with different numbers of latent variables. Overfitting generally occurs when the error rates of the calibration and validation subsets start to diverge when more latent variables are used. The optimal number of latent variables for calibrating a PLS-DA classification model is generally the number at which the lowest error rate results without overfitting.

\section{Methods}

\subsection{Overall Approach}

The research presented in this paper is based on 827 drill core samples that were obtained from exploration drilling into a high-sulphidation epithermal gold-silver 


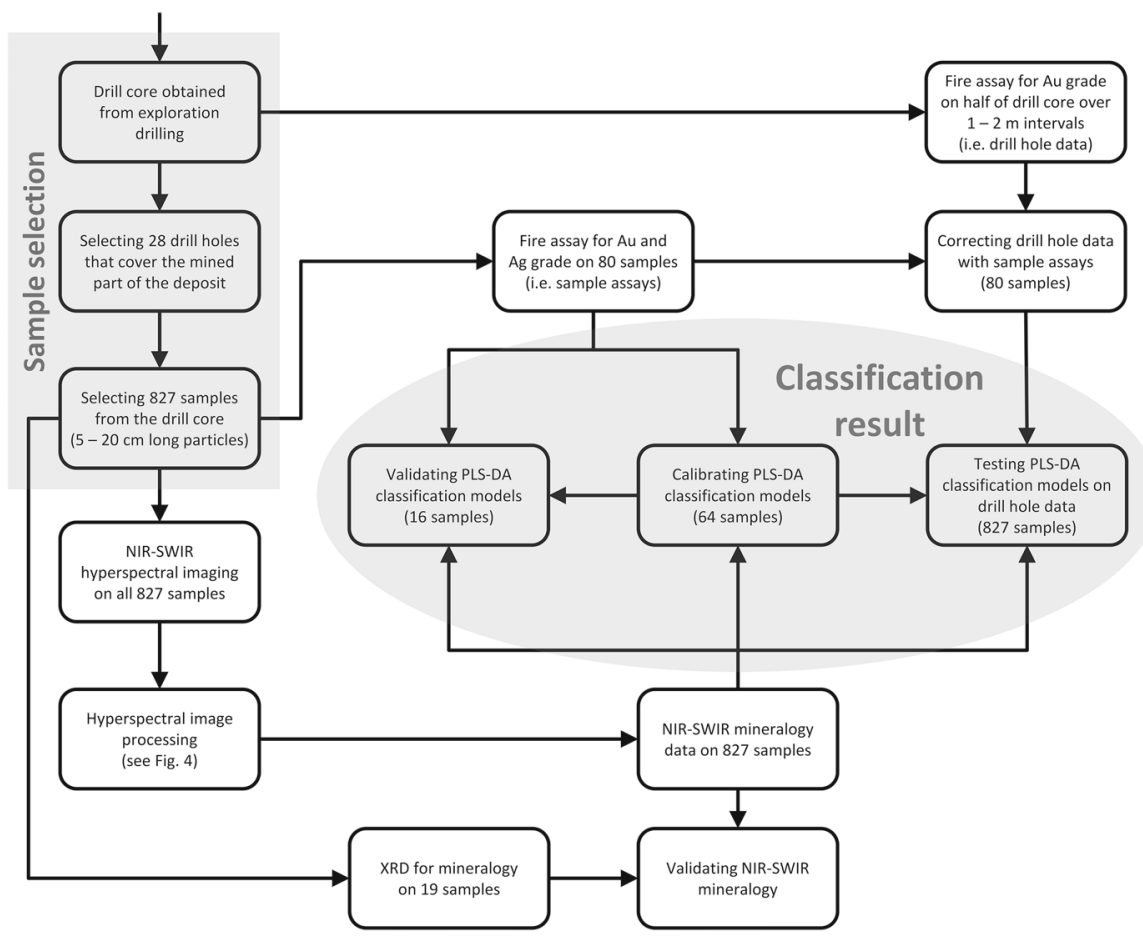

Fig. 2 Overview of the methods used in the study

deposit. These samples are rock particles of about 5-20 $\mathrm{cm}$ long that were selected from half of the splitted drill core of 28 different drill holes. The other half of the splitted drill core was used for geochemical assays by the mining company that exploits the ore deposit and was no longer available at the time of sampling. The drill holes used for sampling were selected in order to cover the spatial extent and variability in lithology and grade of the part of the deposit that will be mined in the future.

Figure 2 presents a schematic overview of the approach that was used to investigate whether NIR-SWIR hyperspectral imaging can be used to discriminate between ore and waste samples. Ore and waste are defined based on a cut-off grade of $0.20 \mathrm{ppm}$ $\mathrm{Au}$, which is realistic for economic processing of the ore on which this study is based. Although some of the ore also contains silver, the silver grade is not considered for defining ore and waste. Most of the material with a relatively low gold grade also has a low silver grade. The different steps shown in Fig. 2 are explained in more detail in the following sections.

\subsection{Acquisition of NIR-SWIR Hyperspectral Images}

NIR-SWIR hyperspectral images of all samples were collected with a SisuROCK instrument (Specim Spectral Imaging Ltd., Oulu, Finland). The images were taken on the cut surface of the splitted drill core samples. Figure 3 shows an example of the 


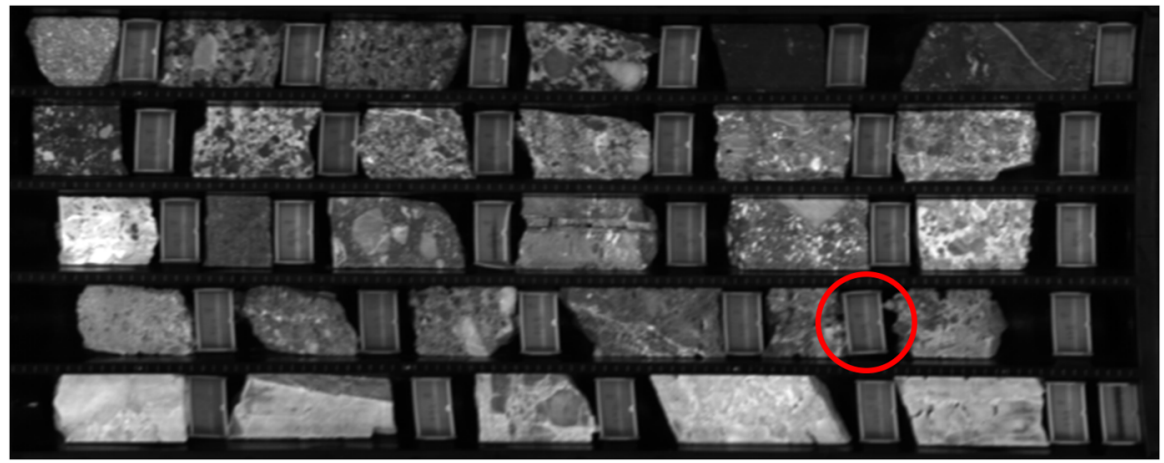

Fig. $31400 \mathrm{~nm}$ band of one of the measured near-infrared (NIR) to short-wavelength infrared (NIR-SWIR) hyperspectral images. The red circle indicates the position of one of the sample labels

$1400 \mathrm{~nm}$ band of one of the hyperspectral images that was collected. The hyperspectral images were recorded on a 380-2500 nm spectral range by using two hyperspectral cameras. The spectral resolution of the images is around $6 \mathrm{~nm}$ on the $380-975 \mathrm{~nm}$ range and around $10 \mathrm{~nm}$ on the $975-2500 \mathrm{~nm}$ range. The pixel size of the images is around $2 \mathrm{~mm}$.

After the NIR-SWIR hyperspectral imagery data were acquired, it was discovered that some of the pixel spectra of the samples are contaminated with absorption features produced by the plastic sample labels within the core trays. These contaminations mainly occur at locations where the sample labels cast a shadow on a sample and are likely produced because the sample labels transmit and/or reflect some radiation before it hits the samples. The position of one of the sample labels is indicated by the red circle in Fig. 3. The absorption effects produced by the labels could not be corrected.

\subsection{Geochemical Data}

Figure 2 shows that different types of geochemical data were used in this study. First of all, fire assays for determining gold grades were performed on 1-2-m long intervals of half of the splitted drill core. The results of these assays were assigned to the samples by using the depth interval from which a sample was taken. This geochemical dataset will be referred to as the drill hole data. It should be noted that the drill hole data are not fully representative, since the samples used in this study are 5-20 cm long, while the interval over which the drill hole data were collected is 1-2 m. Because of this, fire assays were also performed on 80 of the 827 samples after the NIR-SWIR hyperspectral images were acquired. These fire assays included determination of both the gold and silver grade and will be referred to as the sample assays. The drill hole data of the 80 samples on which the sample assays were performed were replaced by the results of these sample assays. Additionally, the mineralogy of 19 samples was determined by X-ray diffraction (XRD) to be able to validate the occurrence of minerals identified from NIR-SWIR hyperspectral imagery. This was also performed after the NIR-SWIR hyperspectral images were acquired. A summary of the different datasets that were used in this study is presented in Table 1. 
Table 1 Overview of the datasets used in the study

\begin{tabular}{llll}
\hline No. of samples & 747 & 61 & 19 \\
\hline $\begin{array}{l}\text { NIR-SWIR hyperspectral } \\
\text { imagery }\end{array}$ & $\mathrm{x}$ & $\mathrm{x}$ & $\mathrm{x}$ \\
Au grade from drill hole data & $\mathrm{x}$ & & \\
$\begin{array}{l}\text { Au and Ag grades from } \\
\text { sample assays }\end{array}$ & $\mathrm{x}$ & $\mathrm{x}$ \\
$\begin{array}{l}\text { Mineralogy from X-ray } \\
\text { diffraction }\end{array}$ & & $\mathrm{x}$ \\
\hline
\end{tabular}

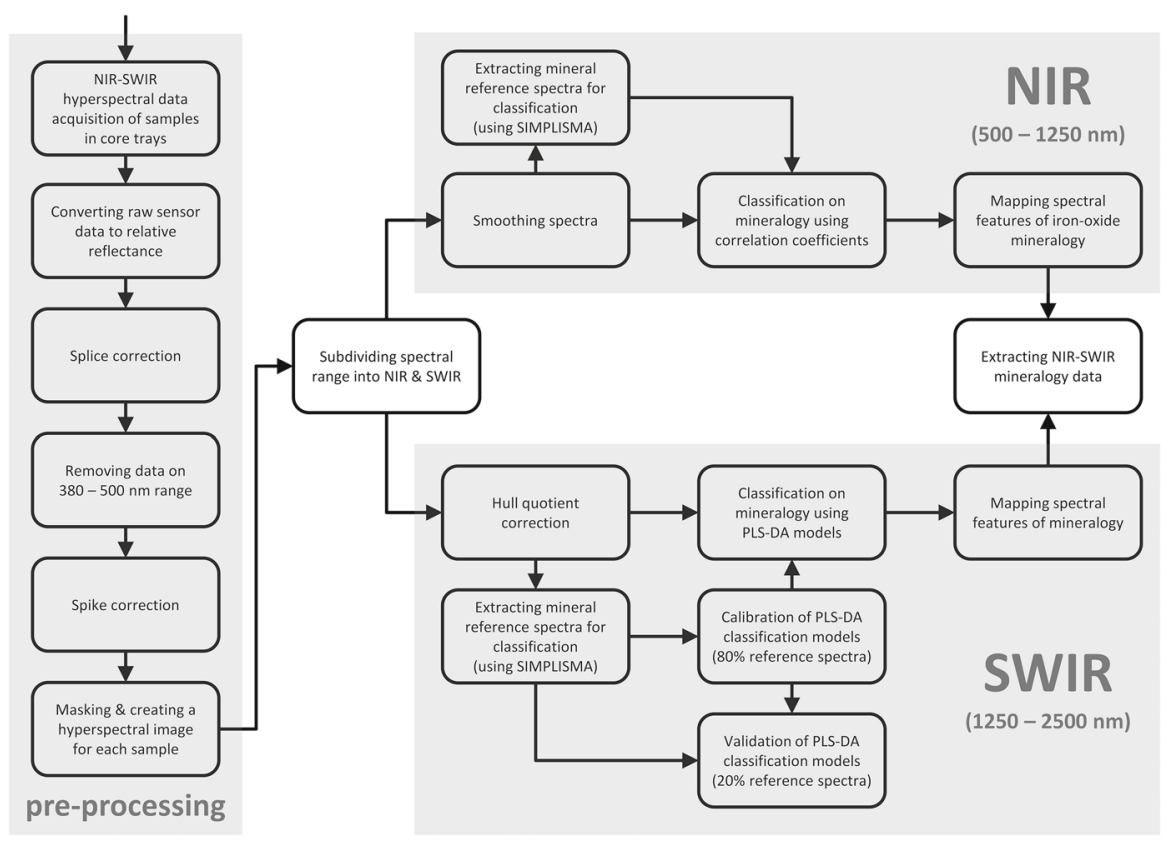

Fig. 4 Overview of NIR-SWIR hyperspectral image processing

\subsection{Image Pre-Processing}

A series of pre-processing steps was required in order to prepare the recorded NIRSWIR hyperspectral data for analysis. These steps are summarised in Fig. 4. The first step that was performed was to convert the recorded data to relative reflections by using a white reference and a dark current calibration.

The second step was to correct for the difference in reflectance that sometimes occurs at the wavelength position where the data from the two detectors are joined. This is commonly referred to as a splice correction. The splice correction was performed by shifting the reflectance values on the $380-975 \mathrm{~nm}$ range to match those on the 975-2500 nm range.

The third pre-processing step that was applied was to remove the data on the $380-500 \mathrm{~nm}$ range. This was performed because the data on this range do not contain 
any characteristic mineral absorption features, while it is relatively noisy due to a low detector sensitivity. The presence of this noisy data without mineralogical information decreases the accuracy of classifying the pixel spectra on mineralogy, which was performed after the pre-processing was completed.

The fourth pre-processing step was a spike correction. Spikes are extremely low or high reflectance values that occur as a result of detector artefacts. Correcting the spikes was also performed to increase the accuracy of the classifications on mineralogy after pre-processing. Spikes were identified by finding local maxima and minima and by applying a threshold to the difference in reflectance that the local maximum or minimum has compared to the surrounding wavelength bands. The threshold that was used was ten times the average reflection difference between consecutive wavelength bands. By using this threshold, no reflectance minima produced by mineral absorptions were incorrectly identified as spikes. However, relatively small spikes still remain in the hyperspectral imagery data. The reflectance values of spikes were removed and replaced with a linear interpolation of the surrounding wavelength bands.

The fifth pre-processing step was masking the hyperspectral images by removing the data of pixels that do not represent a sample. This was performed by manually selecting regions of interest on the image. While selecting the regions of interest, it was attempted to exclude the pixels that are contaminated with absorption features produced by the sample labels as much as possible. However, this was not always successful and, on some samples, these absorption features still occur. A new hyperspectral image for each individual sample was produced from the regions of interest to be able to easily extract the mineralogical properties of every sample after the image processing was completed.

The sixth pre-processing step was to subdivide the spectral range of the NIRSWIR hyperspectral images into an NIR region (500-1250 nm) and an SWIR region (1250-2500 nm). This was done because different types of mineral absorptions take place on these spectral ranges and the accuracy of classifying the pixel spectra on mineralogy increases if these two ranges are treated separately. In the NIR spectral region, the pixel spectra are dominated by absorptions due to electronic transitions of the $\mathrm{Fe}$ ion $\left(\mathrm{Fe}^{2+}\right.$ and $\left.\mathrm{Fe}^{3+}\right)$ that can be used to determine iron-bearing mineralogy. In the SWIR spectral region, the pixel spectra are dominated by absorptions produced by changes in the vibrational energy levels of molecule bonds. These absorptions can be used to detect hydrothermal alteration minerals (AusSpec 2008; Sabins 1999; Thompson et al. 1999).

The final pre-processing step on the NIR range of the hyperspectral images was to smooth the spectra in order to reduce the influence of spectral noise and, thereby, increase the accuracy of the classifications on mineralogy after pre-processing. Smoothing was performed on a window of 11 wavelength bands using a SavitzkyGolay filter with a second-order polynomial (Savitzky and Golay 1964). On the SWIR range of the hyperspectral images, no smoothing was performed since the absorption features on this spectral range are much narrower than those on the NIR range. As a result, smoothing on the smallest possible window removed relatively narrow features with a low absorption intensity.

The final pre-processing step on the SWIR range of the hyperspectral images was to apply a hull quotient correction in order to reduce the influence of absorption effects 

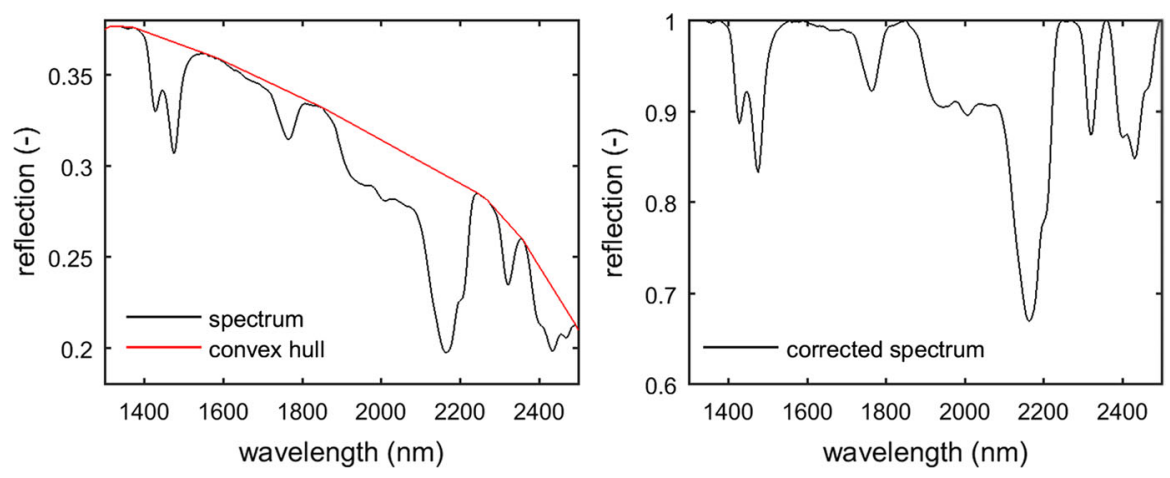

Fig. 5 Example of a hull quotient correction

that are not related to the mineralogy, such as light scattering, particle-size effects, surface roughness and detector artefacts. Removing these absorption effects provides a more robust classification of the pixel spectra on mineralogy. This type of correction was not needed on the NIR hyperspectral images because, on this spectral range, the overall reflectance of the spectrum is dominated by the absorption features of iron oxide. A hull quotient correction is performed by first calculating the convex hull of the spectrum, which represents the shape of the spectrum without the absorption features. The reflectance on each wavelength band of the spectrum is then divided by that of the convex hull to produce the corrected spectrum. The hull quotient correction is illustrated in Fig. 5.

\subsection{Image Processing}

\subsubsection{Mapping Mineralogy from the NIR Hyperspectral Images}

Mapping the mineralogy from the NIR hyperspectral images was mainly focussed on extracting features that represent characteristics of the iron oxide mineralogy. Figure 4 presents an overview of the steps that were taken to achieve this. The first image processing step on the NIR hyperspectral images was to use correlation coefficients to classify the pixels as either pyrite, iron oxide or no diagnostic mineral occurrence. These classifications were based on correlations to 17 pyrite and 389 iron oxide spectra that were selected manually from the measured NIR hyperspectral images. A large number of iron oxide spectra was needed because the variability between these spectra is relatively high. The SIMPLISMA method of Windig and Guilment (1991) was used to extract the purest mineral spectra from the NIR hyperspectral image of each sample to ensure that the variability of the iron oxide spectra throughout the sample set was captured.

Additional to the correlation to pyrite and iron oxide spectra, correlation coefficients were calculated to a spectrum that is similar to iron oxide, but that does not represent iron oxide mineralogy. This was needed to avoid the misclassification of iron oxide, since the similarity between spectra results in relatively high correlation coefficients. It 
is unknown which mineral produces the spectra that are similar to iron oxide. This was also not further investigated since these spectra were observed in the NIR hyperspectral image of only one sample.

The pixel spectra of the NIR hyperspectral images of all samples were classified as pyrite or iron oxide if the correlation coefficient to these spectra was $\geq 0.90$. Manual inspection showed that this threshold provided satisfactory classifications. In case the correlation to both pyrite and iron oxide was $\geq 0.90$, the pixel was classified on the mineral to which the highest correlation coefficient resulted. Pixels were classified as having no diagnostic mineralogy in case the correlation coefficient to pyrite or iron oxide was $<0.90$ or when the correlation to the spectrum used to avoid the misclassification of iron oxide was highest.

The absorption intensity and minimum position of the iron oxide absorption feature that occurs around $900 \mathrm{~nm}$ was automatically calculated for all pixels on which this mineral was classified. The minimum position of this feature usually occurs on a $860-890 \mathrm{~nm}$ range for hematite and on a 910-940 nm range for goethite (AusSpec 2008). It can, therefore, be used to characterise the hematite-goethite mineralogy.

Calculating the absorption intensity and minimum position of the $900 \mathrm{~nm}$ absorption feature of iron oxide was performed by first finding the reflectance maximum on both sides of the absorption feature and then subsetting the spectrum to the spectral range in between both maxima. In case the maximum on the right side of the absorption feature occurs outside the NIR spectral range, the spectrum was subsetted to the spectral range in between the left maximum and $1250 \mathrm{~nm}$. After subsetting, a hull quotient correction was applied and a two-term Gaussian function was fitted to the absorption feature from which the minimum position and the absorption intensity were calculated. Fitting a Gaussian was performed to reduce the influence of spectral noise on the calculated feature properties. The Gaussian function provides a good representation of the shape of absorption features because the physical processes producing these features follow a near-normal distribution (Clark and Roush 1984). The procedure for subsetting, hull quotient correction and fitting a Gaussian is illustrated in Fig. 6 for two example spectra. The minimum position of the iron oxide absorption feature was only calculated if the absorption intensity of the feature was $>0.05$.

\subsubsection{Mapping Mineralogy from the SWIR Hyperspectral Images}

Figure 4 presents an overview of the steps that were taken to map the mineralogy from the SWIR hyperspectral images. The first step was to apply the SIMPLISMA method of Windig and Guilment (1991) in order to extract the purest mineral spectra from the SWIR hyperspectral image of each sample. This method was used to ensure that all the different SWIR-active minerals that occur within the sample set are identified. The SWIR-active mineralogy was determined from the pure spectra by visual inspection and comparison to spectra of the G-MEX spectral interpretation field manual (AusSpec 2008) and the USGS spectral library (Clark et al. 2007).

A set of mineral reference spectra was selected from the pure spectra and additional pixel spectra were added to this set by extracting them manually from the measured SWIR hyperspectral images. The SWIR spectra added were mainly mixed spectra with absorption features of two or more different minerals that were not extracted with 

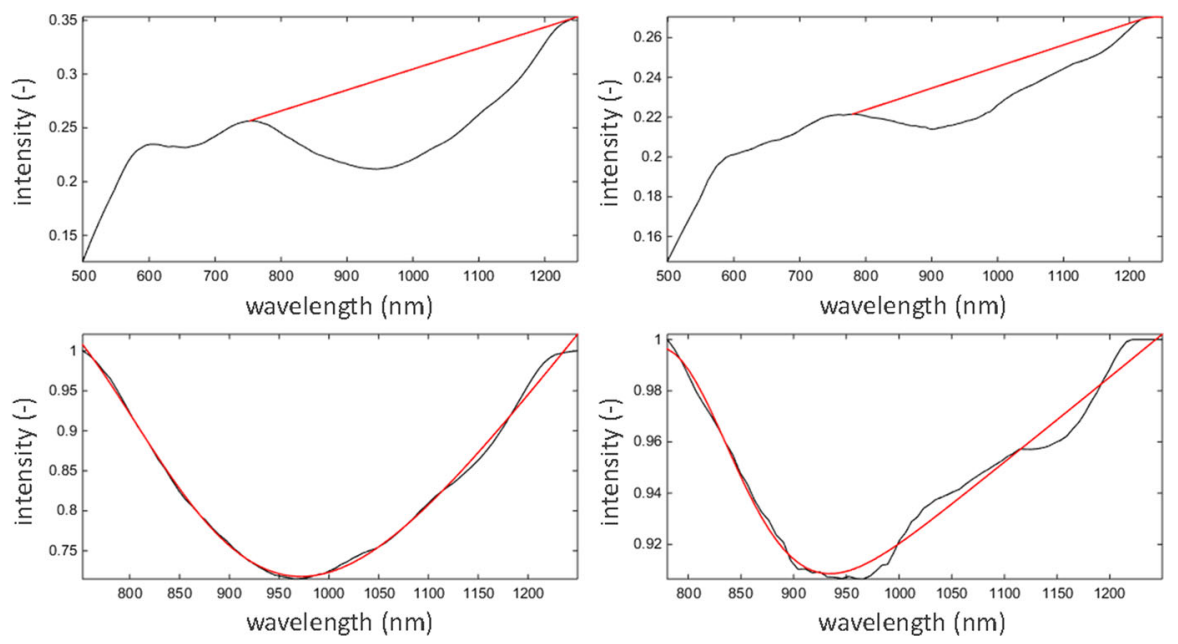

Fig. 6 Two examples of subsetting, hull quotient correction and fitting a Gaussian function to the absorption feature of iron oxide. The two plots at the top present the original spectra on which the convex hull on the spectral range of the absorption feature is represented by the red line. The two plots at the bottom present the subsetted and hull quotient corrected spectra on which the Gaussian fit is represented by the red line

SIMPLISMA. The goal of selecting additional reference spectra was to create a subset of spectra that represents the spectral variability of the SWIR hyperspectral images across all samples as much as possible. For this reason, SWIR spectra containing absorption features of the sample labels, as well as SWIR spectra without any mineral absorption features, were also added to the set of reference spectra.

The total set of reference spectra that was collected consisted of 379 different SWIR spectra, of which the mineralogy was determined through visual inspection. These reference spectra were used to calibrate and validate PLS-DA classification models that allowed the SWIR spectra to be classified on the occurrence or absence of each SWIR-active mineral that was identified. For each individual mineral, a different PLS-DA classification model was produced. The PLS-DA classification models were subsequently used to produce mineral occurrence maps from the SWIR hyperspectral images of all the samples.

To select the optimal number of latent variables for the PLS-DA classifications, $80 \%$ of the SWIR spectra was used to calibrate the models. The error rate of classification versus the number of latent variables was calculated for 1000 randomly chosen calibration and validation subsets. The number of latent variables at which the lowest average error rate resulted for the validation subset was selected for creating the PLS-DA classification model. The response threshold used to perform the classification during cross-validation was based on Bayes' theorem. This means that it is assumed that the responses of each class follow a normal distribution and the chosen threshold is the response value at which those distributions intersect. The PLS-DA was performed with algorithms from the classification toolbox for Matlab from the Milano Chemometrics and QSAR Research Group (Ballabio 2013). Before applying the PLS-DA, the data were centred by setting the mean of each predictor variable to 
zero. This places the average of the data at the origin of the variable space, which ensures that the latent variables describe maximum covariance.

After the pixel spectra of the SWIR hyperspectral images were classified on mineral occurrence, the absorption intensity of several minerals was calculated in order to be able to distinguish between relative mineral abundances. For each mineral, one absorption feature that does not overlap with any of the other minerals was chosen for doing this. Calculating the absorption intensity was performed by subtracting the reflection of the minimum of the absorption feature from that of the baseline of the hull quotient corrected spectrum (reflection of the baseline $=1$ ). Absorption intensities $>0$ were only calculated if the mineral was classified from a pixel spectrum with the corresponding PLS-DA classification model and if the spectrum has a minimum at the wavelength position where the mineral absorption feature should occur. Additionally, the absorption intensity of the water feature that occurs around $1900 \mathrm{~nm}$ was calculated after finding the absorption minimum on the 1850-2100 nm range.

\subsection{Classification of Samples}

Apart from mapping the mineralogy, PLS-DA was also applied to investigate whether the information contained in the mineralogy maps can be used to segment a population of waste samples $(<0.20 \mathrm{ppm} \mathrm{Au})$. Because the drill hole data are not fully representative of the samples, only the samples on which the sample assays were performed were used to calibrate and validate the PLS-DA model for the classification of ore and waste. The drill hole data were only used to further test the performance of the model.

The PLS-DA classifications were based on a data matrix containing the following data for each sample: (i) the relative abundance of each mineral in the mineralogy map ( $\%$ of pixels classified), (ii) the cumulative distribution of the absorption intensity of minerals (\% of pixels > intensity threshold), (iii) the distribution of the minimum position of the absorption feature of iron oxide (\% of pixels within $20 \mathrm{~nm}$ bins) and (iv) the average intensity of the absorption feature of water. Extracting the cumulative distribution of the absorption intensity of minerals was performed by calculating the fraction of pixels with absorption intensities above certain thresholds. The chosen thresholds ranged from 0 to 1 , with increments of 0.05 . Furthermore, the distribution of the minimum location of the iron oxide absorption feature was extracted by first binning the minimum locations and then calculating the fraction of pixels within each bin. The bin size used for calculating this distribution was $20 \mathrm{~nm}$.

The distribution of the minimum position of the iron oxide absorption feature was not cumulative and sums to a constant (i.e. 100\%). This constant sum constraint places restrictions on the correlation structure of the data and can, therefore, influence the outcomes of the PLS-DA (Aitchison 1986). To correct for this, a multiplicative zero replacement followed by a centred log ratio transformation was applied to these data before it was added to the data matrix on which PLS-DA was performed (Aitchison 1986; Martín-Fernández et al. 2003).

The same procedure as with the PLS-DA on SWIR mineral spectra was followed for selecting the optimal number of latent variables and calibrating the PLS-DA model. The only difference was that, before applying the PLS-DA, each predictor variable 
was not only centred, but also scaled by setting the standard deviation to 1 . This was required because the dataset contains different types of variables with different ranges of values, and variables with a relatively high variance will otherwise dominate the model.

\section{Results}

\subsection{Mineralogy Determined from the NIR-SWIR Hyperspectral Images}

Figure 7 presents an overview of the measured NIR and SWIR spectra of all the different minerals that were determined on the samples with NIR-SWIR hyperspectral imagery. All the spectra in this figure resemble those of pure mineral spectra presented in spectral libraries except for diaspore and illite (AusSpec 2008; Clark et al. 2007). The SWIR spectrum of diaspore that is shown in Fig. 7 is mixed with pyrophyllite. The illite spectrum shows a weak shoulder on the left side of the absorptions around 1400 and $2200 \mathrm{~nm}$ that are most likely produced by pyrophyllite or dickite. Spectral mixtures of illite with pyrophyllite as well as illite with dickite were both observed from the SWIR hyperspectral images of the samples. On some samples, spectral mixtures of illite, pyrophyllite and dickite were also observed.

It should be noted that it was not possible to distinguish between spectral mixtures of illite and pyrophyllite and the SWIR spectrum of kaolinite. This is because the absorption features in the mixed spectrum of illite and pyrophyllite overlap with all the absorption features that are produced by kaolinite. Although there are sometimes subtle spectral differences between the spectrum of kaolinite and mixed spectra of pyrophyllite and illite (AusSpec 2008), these were not observed from the measured hyperspectral images. Based on the SWIR hyperspectral images, it is, therefore, unknown if the samples contain kaolinite.

The iron oxide spectrum that is presented in Fig. 7 mainly represents a hematitegoethite mixture. It was usually not possible to distinguish between hematite and goethite from the NIR pixel spectra because these minerals often occur together and their absorption features overlap. For this reason, all the iron oxide spectra were classified as one group. As discussed in Sect. 4.5.1, the exact minimum position of the feature around $900 \mathrm{~nm}$ was calculated to further characterise the iron oxide mineralogy.

The spectrum of water that is shown in Fig. 7 is likely produced by fluid inclusions in quartz. The occurrence of quartz with fluid inclusions is common at epithermal deposits (Simmons et al. 2005). Finally, Fig. 7 shows a spectrum of pyrophyllite that is mixed with the absorption features of a sample label that was in the core tray while acquiring the hyperspectral images. Spectra containing absorption features of the sample labels are found at locations where the sample labels cast shadows on the samples. The absorption features produced by the sample label are the relatively broad features around 1450 and $1700 \mathrm{~nm}$. The mixed spectrum of pyrophyllite and the sample label is presented because it shows that the absorption features of the labels overlap with that of diaspore. This prevented classification of the pixels of the SWIR hyperspectral images on the occurrence of diaspore during the mineral mapping. 

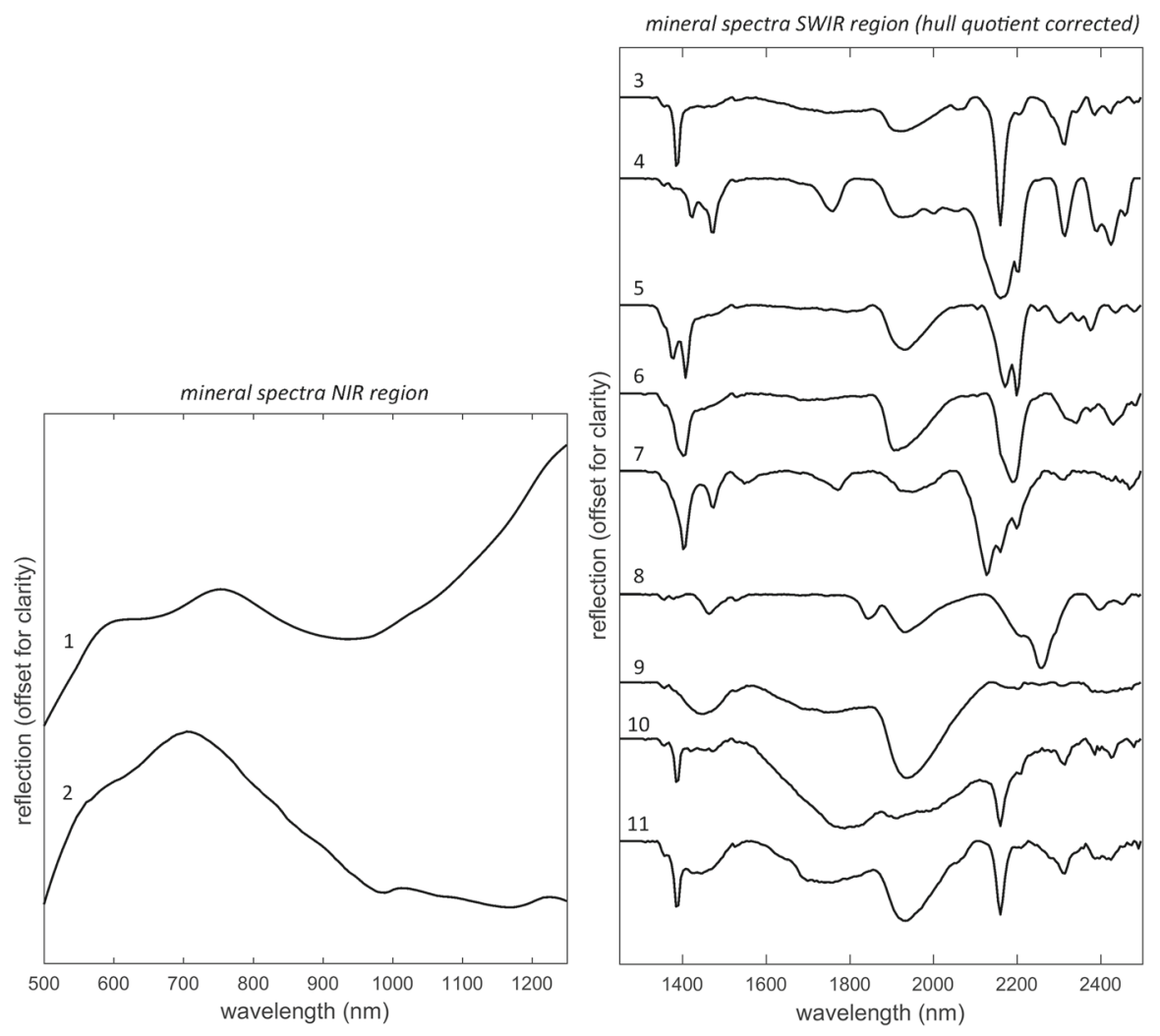

Fig. 7 Examples of measured NIR and SWIR mineral spectra. (1) Iron oxide, (2) pyrite, (3) pyrophyllite, (4) alunite, (5) dickite, (6) illite, (7) zunyite, (8) jarosite, (9) water, (10) diaspore + pyrophyllite, (11) label+ pyrophyllite

The SWIR pixel spectra often contain absorption features of two or three different minerals. From the NIR region of the pixel spectra, only a dominant mineralogy was classified. Some of the NIR and SWIR pixel spectra also contained no characteristic mineral absorptions at all, indicating an absence of NIR- or SWIR-active minerals.

The occurrence of pyrophyllite, alunite, dickite, illite, diaspore and iron oxide was validated by XRD. The occurrence of kaolinite could not be confirmed since the measured XRD spectra did not allow kaolinite and dickite to be distinguished. The occurrence of zunyite, jarosite and pyrite was also not validated by XRD. However, these minerals are known to occur at the deposit from which the samples originate.

\subsection{PLS-DA Classification of SWIR Spectra}

PLS-DA classification models were calibrated on the set of 379 SWIR reference spectra to classify the occurrence of pyrophyllite, alunite, illite-dickite, jarosite and zunyite. Diaspore was not classified because unsatisfactory classification results were obtained due to the overlap of the absorption features of diaspore with those of sample labels. 

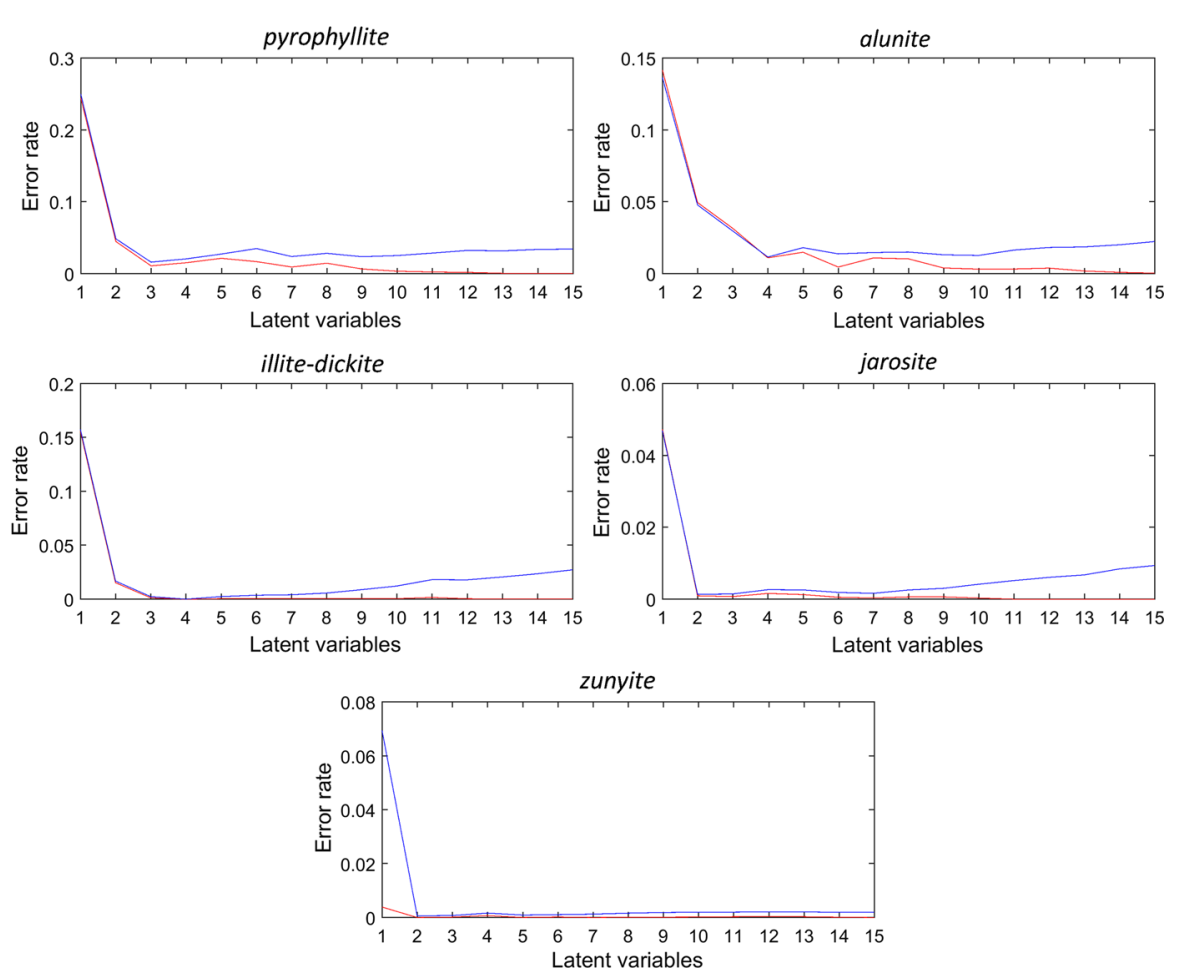

Fig. 8 Error rate versus number of latent variables of the partial least squares discriminant analysis (PLSDA) cross-validation for classification of the mineral reference spectra. The red lines represent the calibration sets and the blue lines the validation sets

Illite and dickite were classified as one group because all the dominant illite absorption features overlap with those of dickite. It was, therefore, not possible to exclude the presence of illite from a spectrum with dickite absorption features. Although illite also produces two secondary absorption features around 2347 and $2440 \mathrm{~nm}$ that do not overlap with dickite (AusSpec 2008), these features were not always observed in the illite spectra due to a relatively low signal-to-noise ratio. Unsatisfactory classification results were also obtained when attempting to distinguish between illite and dickite spectra using PLS-DA. Distinguishing between illite and dickite was performed after the PLS-DA classification by calculating the absorption intensity that each of the minerals produces.

Figure 8 presents the error rates versus the number of latent variables obtained from cross-validating the different PLS-DA classification models. The red lines in this figure represent the calibration sets and the blue lines the validation sets. The number of latent variables that were used to produce the classification models was four for alunite, three for pyrophyllite and illite-dickite, and two for jarosite and zunyite. These numbers were chosen because Fig. 8 shows that the validation subsets have the lowest error rate at these numbers of latent variables. 
Figure 9 presents the responses that resulted from the PLS-DA classifications of mineral spectra. The red colour in this figure represents spectra in which the absorption features of the mineral that is classified were observed by visual inspection. The blue colour represents spectra in which the absorption features of this mineral were not observed. The stars are SWIR spectra that were used for calibrating the classification models and the circles are spectra used for validation. The lines in Fig. 9 represent the thresholds that were used for the classification of spectra. The selected thresholds were the averages between the maximum response of the spectra containing the mineral absorptions and the minimum response of spectra that do not contain the mineral absorptions. In case the responses of both classes overlapped, the spectra with overlapping responses were inspected to investigate if misclassification of these spectra was considered acceptable. Acceptable misclassifications were not included for calculating the classification threshold.

It can be observed from Fig. 9 that there is a clear difference between the responses that were calculated for SWIR spectra that contain the absorption features of each mineral and SWIR spectra that do not. Figure 9 also shows that, for each classification model, the responses of the spectra of the validation set matches the range in responses of the spectra that were used to calibrate the model. This indicates that the PLS-DA classification models produce consistent results. Only for zunyite was a relatively low number of spectra used to produce the classification model and just two spectra could, therefore, be used to validate the classification of zunyite. The reason for this is that spectra with zunyite absorption features were observed on only a few pixel spectra of several samples and not many more zunyite spectra were, therefore, available. However, analysis of the scores and loadings of the PLS-DA models showed that all the classifications, including zunyite, were based on the occurrence of the absorption features of the corresponding mineral.

Figure 9 shows that, for both pyrophyllite and alunite, one spectrum is misclassified by using the response threshold indicated on the figure. For pyrophyllite, inspection of the misclassified spectrum showed that this is a mixed spectrum of pyrophyllite and illite that is relatively noisy. The reason that this spectrum is misclassified is likely due to the noise and the fact that the mixed pyrophyllite-illite spectrum is similar to the spectrum of dickite. Since all the other pyrophyllite spectra were correctly classified, misclassification of a single noisy spectrum was considered acceptable. The alunite spectrum that is misclassified is a mixed spectrum of dickite and alunite in which the dickite absorptions features dominate. Alunite was identified from the occurrence of only one absorption feature with a relatively low intensity. This indicates that, if alunite was correctly identified from the SWIR spectrum by visual inspection, it is present in only minor occurrences. Misclassification of this spectrum by the PLS-DA model was, therefore, also considered acceptable.

\subsection{Mineralogy Mapping}

Figure 10 presents the results of mapping the mineral absorption intensity from the hyperspectral images of six samples. For the SWIR-active minerals, absorption intensities $>0$ were only mapped if the mineral that produces the absorption feature was 

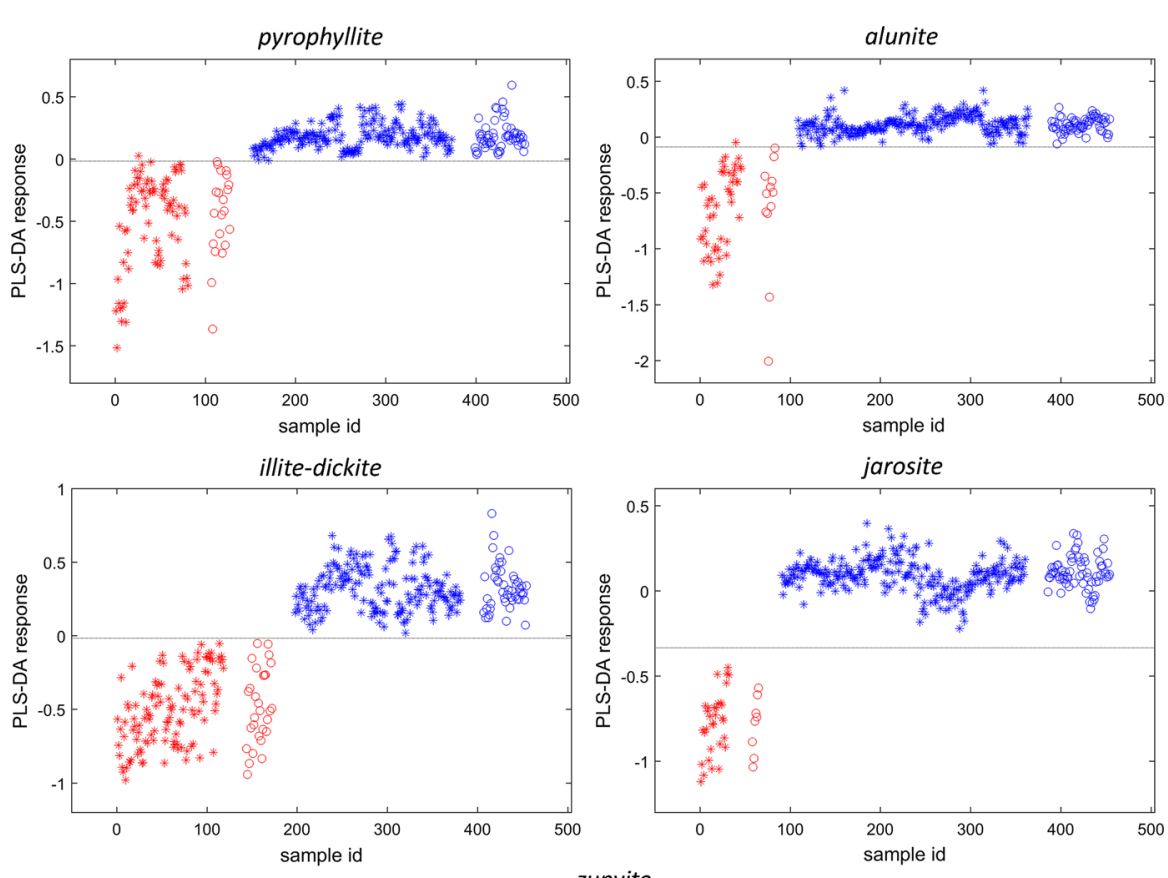

zunyite

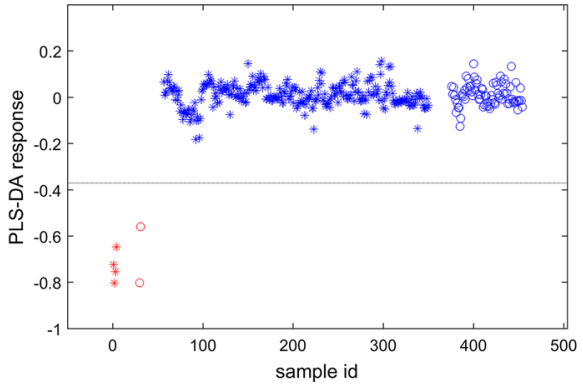

Fig. 9 Calculated responses of the PLS-DA classifications of the 379 mineral reference spectra. The red colour represents spectra containing absorptions of the associated mineral. The blue colour represents spectra that do not contain absorptions of the associated mineral. The stars represent spectra used for calibrating the model and the circles represent spectra used for validation

classified from a pixel spectrum with the corresponding PLS-DA classification model. For iron oxide, the absorption intensity and mineral position was mapped if the correlation coefficient between the pixel spectrum and that of one of the iron oxide spectra was $>0.9$ and higher than that of pyrite or the unknown spectrum. The intensity of the water feature was mapped for all pixel spectra.

The absorption intensity of jarosite and zunyite was not characterised because these minerals occur on only a few samples. The absorption intensity of alunite, pyrophyllite, illite-dickite and dickite shown in Fig. 10 is that of one of the absorption features which each mineral produces around $1400 \mathrm{~nm}$. These absorption features were chosen because these are relatively intense and have the least overlap with absorption features 

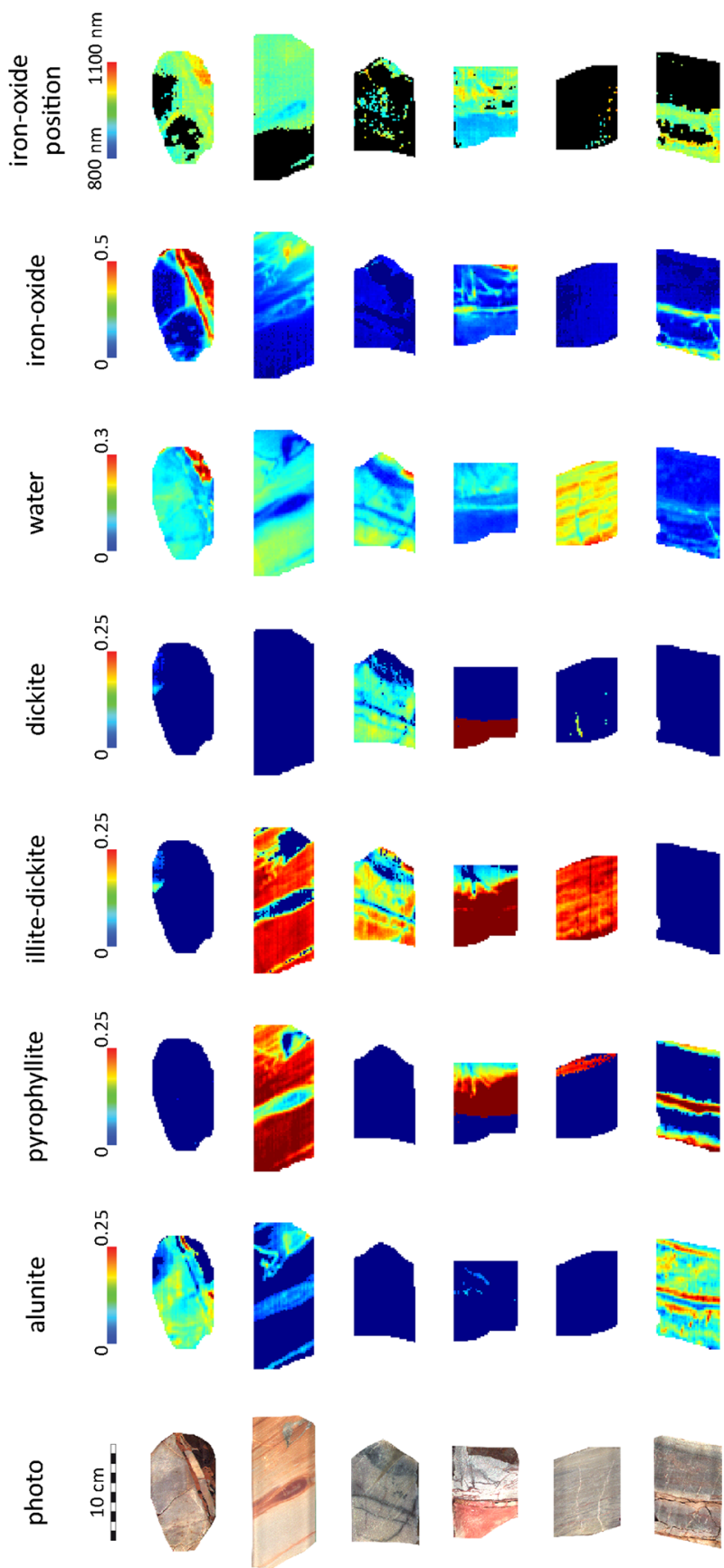

$\hat{-1}$

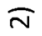

$\bar{m}$

ซ

เก

6

Fig. 10 Result of mapping the absorption intensity of alunite, pyrophyllite, illite-dickite, water and iron oxide and the minimum position of the iron oxide absorption feature from the NIR-SWIR hyperspectral images 
of the other minerals. Since all the absorption features of illite overlap with dickite, the absorption intensity of illite alone could not be characterised. The occurrence of illite can be inferred from the absence of the dickite absorption while the illite-dickite absorption is present. In Fig. 10, this is the case for sample 5 for example.

It was mentioned in Sect. 5.1 that, due to overlapping features, it was not possible to distinguish between spectral mixtures of illite and pyrophyllite and the SWIR spectrum of kaolinite. This means that, if absorption intensities $>0$ result for both pyrophyllite and illite, it is possible that these pixels actually represent kaolinite. In Fig. 10, this is the case for sample 2 and partly for sample 4 .

It was explained in Sect. 4.5.1 that the $900 \mathrm{~nm}$ iron oxide absorption usually occurs on a 860-890 $\mathrm{nm}$ range for hematite and on a 910-940 $\mathrm{nm}$ range for goethite (AusSpec 2008). However, Fig. 10 shows that the minimum positions of the $900 \mathrm{~nm}$ iron oxide absorption in the measured NIR hyperspectral images occur on a 900-1050 nm range. The difference between the minimum positions reported by AusSpec (2008) and those calculated from the measured NIR hyperspectral images likely results from the way the minimum positions were calculated. The minimum positions of the absorption features of iron oxide minerals reported by AusSpec (2008) are based on uncorrected spectra, while on the measured iron oxide spectra, a hull quotient correction was applied in order to calculate the absorption intensity. Since the maximum on the left side of the absorption feature usually has a lower intensity than the maximum on the right side of the feature, a hull quotient correction will shift the minimum position to longer wavelengths. However, this shift in minimum positions will affect all the iron oxide spectra in the same way and the calculated minimum positions, therefore, still represent differences in the hematite-goethite mineralogy.

Figure 10 shows that mapping the minimum position of the $900 \mathrm{~nm}$ iron oxide absorption reveals additional differences between samples. For example, sample 1 in Fig. 10 contains regions where the minimum position of the iron oxide absorption occurs at relatively long wavelengths. The minimum positions at these wavelengths cannot be observed in the image of sample 2. The iron oxide that occurs at the surface of sample 1 is, therefore, more similar to goethite compared to that of sample 2 .

\subsection{Classification of Ore and Waste}

Table 2 presents an overview of the mineralogical data on which the PLS-DA classification of ore and waste samples was performed. These data were extracted from the mineralogy maps of each sample. Several examples of these maps were presented in Fig. 10. The pixel fractions of zunyite were not used in the PLS-DA classification of ore and waste because zunyite was classified on only three samples with a maximum of $0.7 \%$ of the pixels.

Figure 11 presents the average error rate versus the number of latent variables that are obtained from 1000 cross-validations of the PLS-DA classification of ore and waste samples. The red line in this figure represents the calibration set and the blue line the validation set. Figure 11 shows that the error rate of the validation set only increases when more than one latent variable is used. Because this indicates that the PLS-DA model is overfitting, one latent variable was chosen for producing the PLS-DA classification of ore and waste. 
Table 2 Overview of data used in the partial least squares discriminant analysis (PLS-DA) classification of ore and waste samples

\begin{tabular}{lllll}
\hline & $\begin{array}{l}\text { Mineral abundance } \\
\text { (\% pixels } \\
\text { classified) }\end{array}$ & $\begin{array}{l}\text { Absorption intensity } \\
\text { distribution }(\% \\
\text { pixels }>\text { threshold })\end{array}$ & $\begin{array}{l}\text { Distribution of } \\
\text { minimum position } \\
\text { (\% pixels in bins) }\end{array}$ & $\begin{array}{l}\text { Average } \\
\text { absorption } \\
\text { intensity }\end{array}$ \\
\hline Pyrophyllite & $\mathrm{x}$ & $\mathrm{x}$ & & \\
Alunite & $\mathrm{x}$ & $\mathrm{x}$ & & \\
Illite-dickite & $\mathrm{x}$ & $\mathrm{x}$ & & \\
Dickite & $\mathrm{x}$ & $\mathrm{x}$ & & \\
Jarosite & $\mathrm{x}$ & & $\mathrm{x}$ & $\mathrm{x}$ \\
Pyrite & $\mathrm{x}$ & $\mathrm{x}$ & &
\end{tabular}

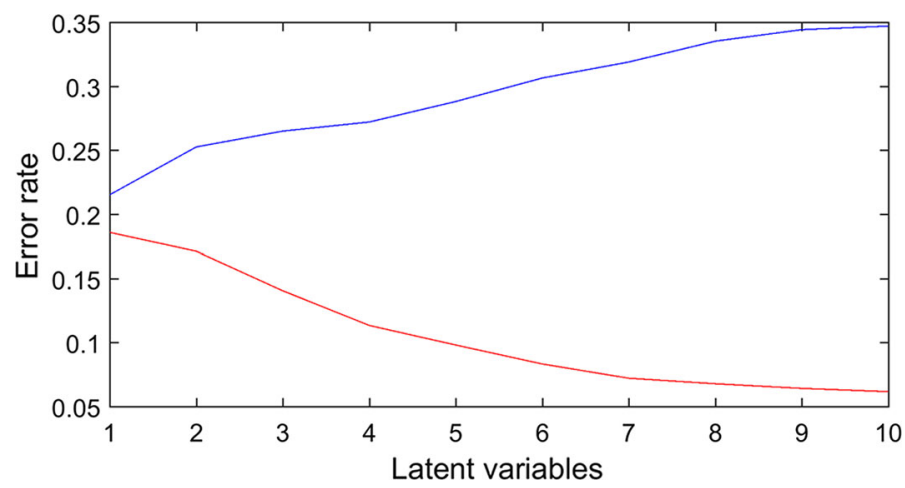

Fig. 11 Error rate versus number of latent variables of the PLS-DA cross validation for the classification of ore and waste samples. The red line represents the calibration set and the blue line the validation set

Figure 12 presents the PLS-DA responses of the classification in which the ore-waste designation was based on the gold grade from the sample assays. The blue colour in this figure represents waste samples and the red colour ore samples. The stars are samples that were used for calibrating the PLS-DA classification model and the circles are samples used for validation. It can be observed from Fig. 12 that the calculated responses of the validation sets match the range in responses of the calibration sets. This indicates that the PLS-DA classification model produces consistent results.

It can be observed from Fig. 12 that the PLS-DA classification model was not able to fully separate ore from waste. This is likely because some of the waste samples in the samples set have an SWIR-active mineralogy that is similar to some of the ore samples. This was also expected since it is unlikely that there is a discrete difference in alteration mineralogy at some arbitrary cut-off on the gold grade. However, Fig. 12 also shows that, for most of the waste samples, a higher response is calculated than for most of the ore samples. The PLS-DA responses can, therefore, be used to predict whether a sample is more likely to be waste or ore. 


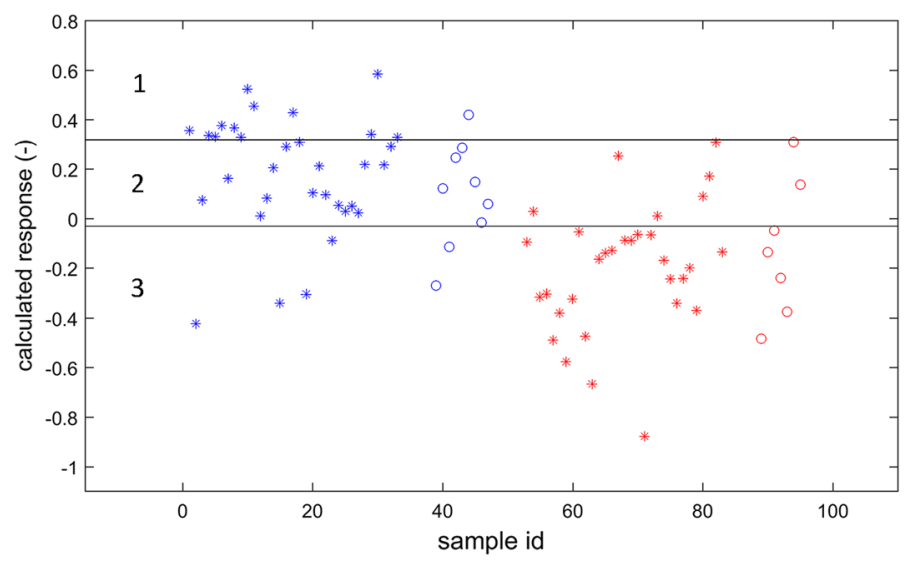

Fig. 12 Calculated PLS-DA responses of ore and waste samples. Ore-waste designation is based on the gold grade from the sample assays. The blue colour represents waste samples. The red colour represents ore samples. The stars represent samples used for calibrating the model and the circles represent samples used for validation

Table 3 Results of the PLS-DA classification of ore and waste

\begin{tabular}{lcccccccc}
\hline & \multicolumn{3}{c}{ Based on 80 sample assays } & & \multicolumn{3}{c}{ Based on 827 drill hole assays ${ }^{\mathrm{a}}$} \\
\cline { 2 - 3 } & Group 1 & Group 2 & Group 3 & & Group 1 & Group 2 & Group 3 \\
\hline Fraction of samples (\%) & 16.3 & 38.7 & 45.0 & & 7.5 & 45.3 & 47.2 \\
Waste samples in group (\%) & 100.0 & 74.2 & 16.7 & & 91.9 & 70.4 & 29.2 \\
$\mathrm{Au}(\mathrm{ppm})$ minimum & 0.00 & 0.00 & 0.00 & & 0.00 & 0.00 & 0.00 \\
$\mathrm{Au}(\mathrm{ppm})$ maximum & 0.20 & 0.96 & 14.68 & & 1.74 & 31.22 & 22.39 \\
$\mathrm{Au}(\mathrm{ppm})$ average & 0.06 & 0.19 & 1.51 & & 0.08 & 0.48 & 1.15 \\
$\mathrm{Ag}(\mathrm{ppm})$ minimum & 0.00 & 0.00 & 0.00 & & & \\
$\mathrm{Ag}(\mathrm{ppm})$ maximum & 8.00 & 11.10 & 33.55 & & & \\
$\mathrm{Ag}(\mathrm{ppm})$ average & 0.62 & 0.56 & 4.24 & & & \\
\hline
\end{tabular}

${ }^{a}$ The drill hole data are not fully representative, since the samples used in this study are 5-20 $\mathrm{cm}$ long, while the interval over which the drill hole data were collected is $1-2 \mathrm{~m}$

The two lines in Fig. 12 are the two thresholds chosen for the classification of samples. The threshold at 0.32 was chosen in order to segment a group of samples that are all waste and the threshold at -0.03 was chosen to segment a group that consists mainly of ore samples. Although segmenting ore samples was not one of the objectives of the study, this was performed to illustrate the potential of using PLS-DA classification models to distinguish between gold grades. Table 3 presents the resulting gold and silver grades when the responses and thresholds shown in Fig. 12 are used to classify the samples. The group numbers in this table refer to the numbers annotated on Figs. 12 and 13. It can be observed from Table 3 that significant differences between the average gold grades of the three groups result. The silver grade of group 3 is also significantly higher than that of the other two groups. 


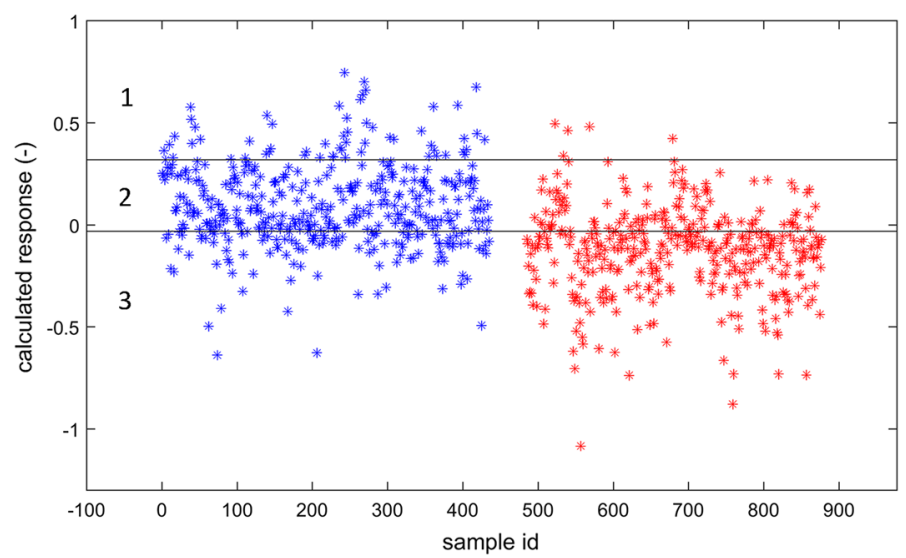

Fig. 13 Calculated PLS-DA responses of all ore and waste samples. Ore-waste designation is based on the gold grade from the drill hole data. The blue colour represents waste samples. The red colour represents ore samples

Figure 13 presents the responses of all samples that were calculated by using the same PLS-DA classification model used for the classification presented in Fig. 12. The lines shown in this figure also represent exactly the same thresholds as those in Fig. 12. The red and blue colours refer, in this case, to the ore-waste designation that is based on the gold grades from the drill hole data. The ore-waste designation of the samples that were used to calibrate the PLS-DA model is still the same, since the drill hole data of samples on which sample assays were performed were replaced by the results of these assays. As explained previously, the drill hole data are not fully representative, since the samples used in this study are 5-20 cm long, while the interval over which the drill hole data were collected is 1-2 $\mathrm{m}$. The ore-waste designations that are shown in Fig. 13 might, therefore, not be completely correct.

The responses in Fig. 13 show a similar trend to those in Fig. 12. Table 3 presents the resulting gold grades from the drill hole data when the responses and thresholds shown in Fig. 13 are used to classify the samples. This table shows that, based on the drill hole data, a population consisting of only waste samples is no longer segmented. However, it also shows that the average gold grade of the group of waste samples (group 1) is still significantly lower than the economic cut-off grade of $0.20 \mathrm{ppm}$ $\mathrm{Au}$. Furthermore, there are also still significant differences between the average gold grades of the three groups. The drill hole data, therefore, validates that it is possible to use NIR-SWIR hyperspectral imagery to distinguish between the gold grades of the samples. The differences in silver grade could not be compared, since no silver grades were available from the drill hole data.

Figure 14 presents the loadings of the latent variable that was used in the PLS-DA classification of ore and waste samples. The annotated numbers on Fig. 14 refer to the different types of variables used in the classification. The cumulative distribution of the absorption intensity of pyrophyllite, alunite, dickite, illite-dickite and iron oxide are represented by the numbers 1, 2, 3, 4 and 5, respectively. In these distributions, the absorption intensity increases from left to right. Number 6 represents the distribution of 


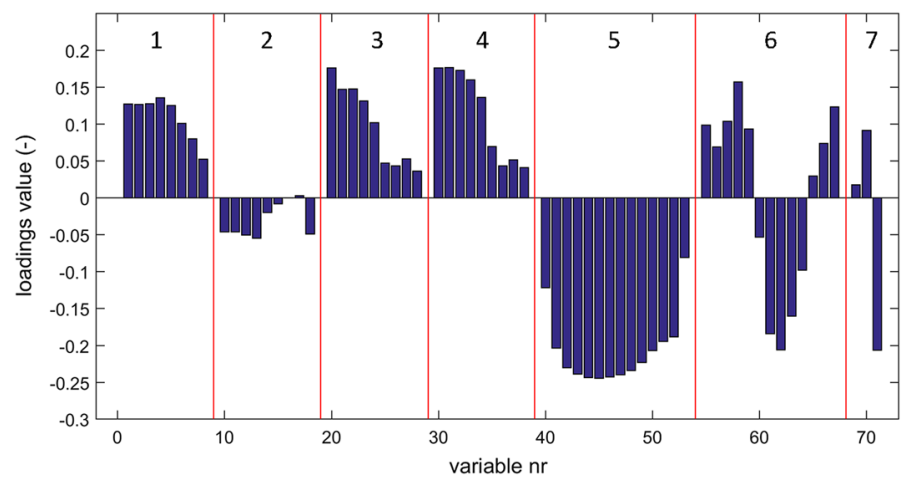

Fig. 14 PLS-DA loadings of the classification of ore and waste samples. 1-5 Absorption intensity distribution of pyrophyllite, alunite, dickite, illite-dickite and iron oxide. 6 Distribution of the minimum position of the iron oxide absorption. 7 Fraction of pyrite pixels, fraction of jarosite pixels and the average intensity of the water absorption feature

the minimum position of the iron oxide absorption feature with increasing wavelength from left to right. The variables represented by number 7 are the fractions of pyrite and jarosite pixels and the average intensity of the water absorption feature (from left to right).

Analysis of the sample scores on the latent variable used in the PLS-DA classification model showed that the waste samples have relatively high score values. This means that waste samples are associated with variables that have high loadings values. It can be observed from Fig. 14 that pyrophyllite, dickite and illite have high loadings values, and a relatively high abundance of these minerals in the mineralogy maps is, therefore, a strong indicator of waste. Regarding dickite and illite, Fig. 14 also shows that mainly low intensity absorptions of these minerals indicate waste. Furthermore, Fig. 14 shows that relatively high fractions of jarosite pixels are more associated with waste samples. However, the fact that the loadings value of jarosite is not very high does suggest that it is not such a strong indicator of waste as pyrophyllite, dickite or illite. This could also be related to the fact that not many samples contain jarosite.

It can be observed from Fig. 14 that a relatively high abundance of iron oxide is a strong indicator of ore. The loadings show that this mainly concerns iron oxide with minimum positions in the ranage $950-1050 \mathrm{~nm}$. Furthermore, it can be derived from Fig. 14 that ore samples are associated with relatively intense water features and ore samples can contain alunite. Finally, Fig. 14 shows that the pixel fractions of pyrite have a very low loadings value and, therefore, do not have a large influence on the classifications.

\section{Discussion}

Table 3 showed that NIR-SWIR hyperspectral imagery could be used to segment a population of waste samples from the set of 80 samples on which the sample assays 
were performed. This population represents $31 \%$ of all the waste samples in this sample set. Additional research is still required to investigate whether removing this fraction of waste with sensor-based sorting equipment is economically feasible. This will depend on the costs of sorting, the costs of conventional processing, the efficiency of the sorting machine, the amount of waste that is rejected and the value of the gold that is contained by the waste. Economic benefits are generally provided if the value of the amount of gold and silver that are lost by discarding material do not exceed the difference in costs between sensor-based sorting and conventional ore processing. Table 3 shows that $16.3 \%$ of the samples can be rejected at an average gold grade of $0.06 \mathrm{ppm}$. This is a significant amount of material at a gold grade that is less than half of the economic cut-off grade of $0.20 \mathrm{ppm}$. Since sensor-based sorting is generally cheaper than conventional processing methods (Buxton and Benndorf 2013; Lessard et al. 2014; Salter and Wyatt 1991; Sivamohan and Forssberg 1991; Wills and Finch 2016), this means there is significant potential that removing this waste with sensorbased sorting can provide economic benefits.

Table 3 shows that, when all the samples are classified with the PLS-DA model, group 1 represents $7.5 \%$ of the sample set. This is a significant difference compared to the $16.3 \%$ of the samples that were classified into group 1 for the sample set on which the sample assays were performed. Furthermore, only $13 \%$ of the total amount of waste samples was segmented when the PLS-DA model was applied to all samples. This indicates that the set of samples on which the sample assays were performed does not fully represent the mineralogical variability of the entire sample set. In order to obtain a more representative result, additional geochemical assays would be required. This was not performed due to limited funding. However, the ability to reject $7.5 \%$ of the mined material at an average grade of $0.08 \mathrm{ppm} \mathrm{Au} \mathrm{still} \mathrm{indicates} \mathrm{that} \mathrm{there}$ is potential that removing waste with sensor-based sorting can provide economic benefits.

The different mineral assemblages that were observed from the NIR-SWIR hyperspectral images are characteristic for different zones of hydrothermal alteration within epithermal deposits (Sillitoe 1993; Simmons et al. 2005; White and Hedenquist 1995). The ability to segment a population of waste samples is, therefore, based on a relationship between gold mineralisation and the formation of alteration minerals. It resulted that detecting occurrences of pyrophyllite, diaspore, dickite and/or illite can be used as indicators of waste. Ore is associated with iron oxide, alunite and intense water features that are most likely produced by fluid inclusions within quartz. Figure 1 showed that the main ore zone at high-sulphidation epithermal deposits is closest to the epithermal veins. This figure also shows that, further away from the veins, the alteration mineralogy gradually changes from a vuggy quartz to quartz-alunite to dickite/kaolinite-pyrophyllite to smectite/mixed layer clay. Since this means that occurrences of pyrophyllite, dickite and illite are further away from the main ore zone, this validates that these minerals can be used as indicators of waste. The reason that alunite is sometimes still associated with ore samples can be explained by the fact that this mineral is deposited closer to the epithermal veins (Sillitoe 1993; Simmons et al. 2005).

It was discussed in Sect. 5.1 that it was not possible to distinguish between SWIR spectral mixtures of illite and pyrophyllite and the SWIR spectrum of kaolinite. This 
is because the absorption features in the mixed spectrum of illite and pyrophyllite overlap with all the absorption features produced by kaolinite. Figure 14 showed that the lowest error rate for the validation set results if the PLS-DA model uses one latent variable and the PLS-DA loadings of this latent variable show that both illite and pyrophyllite are associated with waste samples. This indicates that, if kaolinite actually occurs, it is likely also associated with waste samples. This is because if this is not the case, it would be expected that a lower error rate can be achieved if the PLS-DA was performed with additional latent variables that distinguish between pure and mixed occurrences of pyrophyllite and illite.

PLS-DA proved to be a relatively robust method for mapping mineral occurrences from the pixel spectra of SWIR hyperspectral images. This is because the PLS-DA models can be cross-validated to assess the classification performance. Furthermore, the scores and loadings can be inspected to validate that the classifications are based on the absorption features of the minerals that are classified. Using PLS-DA for mineral mapping does require a sufficiently large set of pre-classified spectra that can be used to calibrate the classification models. It is also important that cross-validation is carried out properly to ensure that the PLS-DA classification models are not overfitting.

Compared to mineral mapping techniques based on spectral similarity (e.g. correlation coefficients or spectral angle mapping), PLS-DA also provides the advantage that it is possible to classify mineral occurrence from a variety of mixed mineral spectra. When using similarity-based techniques, a different class needs to be assigned to each different mixture of SWIR-active minerals in order to be able to distinguish them from each other. This increases the risk of misclassification when a large variety of SWIR-active mineral mixtures occurs that have relatively subtle spectral differences.

PLS-DA also proved to be an effective method to investigate the possibilities of using the information contained in the mineralogy maps to classify samples. This method has the advantage that it is relatively easy to identify the mineralogical differences on which classifications are based by inspecting the PLS-DA scores and loadings. Furthermore, the responses are calculated in such a way that the relative difference between the responses of the two classes is maximised. This means that, when it is not possible to completely separate the classes, a threshold on the calculated responses can sometimes be selected that allows a fraction of the samples from one of the classes to be separated without including samples of the other class. This is especially helpful when investigating solutions for sensor-based sorting of ore and waste, since misclassification of the ore is usually not desired, while it is often sufficient if only a fraction of the waste material can be segmented. It would be possible to further refine the classification of waste by producing new classification models that are calibrated to discriminate only the fraction of waste samples that could be segmented in an initial PLS-DA classification. This was not performed here, since the initial PLS-DA model already satisfied the aim of this study.

\section{Conclusions}

Near-infrared to short-wavelength infrared (NIR-SWIR) hyperspectral imagery could be used to segment a population of waste samples from the drill core samples obtained 
from a high-sulphidation epithermal gold-silver deposit. This is based on detecting occurrences of pyrophyllite, dickite and/or illite, which are commonly deposited further away from the ore-bearing epithermal veins, while the absence of SWIR-active minerals or detected occurrences of alunite are more closely associated with these structures.

The ability to identify waste with NIR-SWIR spectral sensors means there is potential that sensor-based sorting can be used to remove this waste from mineral processing operations and provide economic benefits. However, additional research is still required to assess the economic feasibility of such a sensor-based sorting application. The largest uncertainty is the extent to which the results of this study can be reproduced with sensor-based sorting equipment on run-of-mine material. It is recommended to investigate this by performing pilot-scale tests with a sensor-based sorting machine.

Partial least squares discriminant analysis (PLS-DA) proved to be an effective method for mapping the mineralogy from the SWIR hyperspectral images, as well as investigating the possibilities of using the information contained in the mineralogy maps to segment a population of waste samples.

Acknowledgements The authors would like to thank Barrick Gold for providing test samples and funding for this research.

Open Access This article is distributed under the terms of the Creative Commons Attribution 4.0 International License (http://creativecommons.org/licenses/by/4.0/), which permits unrestricted use, distribution, and reproduction in any medium, provided you give appropriate credit to the original author(s) and the source, provide a link to the Creative Commons license, and indicate if changes were made.

\section{References}

Abdi H (2010) Partial least squares regression and projection on latent structure regression (PLS Regression). Wiley Interdiscip Rev Comput Stat 2:97-106

Aitchison J (1986) The statistical analysis of compositional data. Chapman and Hall, London

AusSpec (2008) G-MEX: guides for mineral exploration: spectral interpretation field manual. AusSpec International Ltd., Queenstown

Ballabio D (2013) Classification toolbox. Available online at: http://michem.disat.unimib.it/chm/downloa d/softwares.htm. Accessed Jan 2015

Buxton MWN, Benndorf J (2013) The use of sensor derived data in optimization along the Mine-ValueChain. In: Proceedings of the 15th International ISM Congress, Aachen, Germany, September 2013, pp 324-336

Clark RN (1999) Spectroscopy of rocks and minerals, and principles of spectroscopy. Man Remote Sens $3: 3-58$

Clark RN, Roush TL (1984) Reflectance spectroscopy: quantitative analysis techniques for remote sensing applications. J Geophys Res Solid Earth 89:6329-6340

Clark RN, Swayze GA, Wise RA, Livo KE, Hoefen TM, Kokaly RF, Sutley SJ (2007) USGS digital spectral library splib06a. U.S. Geological Survey Digital Data Series 231. https://speclab.cr.usgs.gov/spectral .lib06/ds231/. Accessed Jan 2012

Dalm M, Buxton MWN, van Ruitenbeek FJA, Voncken JHL (2014) Application of near-infrared spectroscopy to sensor based sorting of a porphyry copper ore. Miner Eng 58:7-16. https://doi.org/10.10 16/j.mineng.2013.12.016

Dalm M, Buxton MWN, van Ruitenbeek FJA (2017) Discriminating ore and waste in a porphyry copper deposit using short-wavelength infrared (SWIR) hyperspectral imagery. Miner Eng 105:10-18

Hollas JM (2004) Modern spectroscopy. Wiley, Chichester 
Hunt GR (1977) Spectral signatures of particulate minerals in the visible and near infrared. Geophysics 42:501-513

Lessard J, de Bakker J, McHugh L (2014) Development of ore sorting and its impact on mineral processing economics. Miner Eng 65:88-97. https://doi.org/10.1016/j.mineng.2014.05.019

Martín-Fernández JA, Barceló-Vidal C, Pawlowsky-Glahn V (2003) Dealing with zeros and missing values in compositional data sets using nonparametric imputation. Math Geol 35:253-278

Næs T, Martens H (1984) Multivariate calibration. II. Chemometric methods. TrAC Trends Anal Chem 3:266-271

Pirajno F (1992) Hydrothermal mineral deposits: principles and fundamental concepts for the exploration geologist. Springer, Berlin

Sabins FF (1999) Remote sensing for mineral exploration. Ore Geol Rev 14:157-183

Salter JD, Wyatt NPG (1991) Sorting in the minerals industry: past, present and future. Miner Eng 4:779-796

Savitzky A, Golay MJ (1964) Smoothing and differentiation of data by simplified least squares procedures. Anal Chem 36:1627-1639

Sillitoe RH (1993) Epithermal models: genetic types, geometrical controls and shallow features. In: Kirkham RV, Sinclair WD, Thorpe RI, Duke JM (eds) Mineral deposit modeling. Geological Association of Canada Special Paper 40:403-417

Simmons SF, White NC, John DA (2005) Geological characteristics of epithermal precious and base metal deposits. Econ Geol 100:485-522

Sivamohan R, Forssberg E (1991) Electronic sorting and other preconcentration methods. Miner Eng 4:797-814

Sjöström M, Wold S, Söderström B (1986) PLS discriminant plots. Pattern Recognit Pract II:461-470

Thompson AJB, Hauff PL, Robitaille AJ (1999) Alteration mapping in exploration: application of shortwave infrared (SWIR) spectroscopy. SEG Newsl 39:16-27

White NC, Hedenquist JW (1995) Epithermal gold deposits: styles, characteristics and exploration. SEG Newsl 23:9-13

Wills BA, Finch JA (2016) Chapter 14—sensor-based ore sorting. In: Wills' mineral processing technology (8th edn). Butterworth-Heinemann, Boston, pp 409-416. http://dx.doi.org/10.1016/B978-0-08-0970 53-0.00014-5

Windig W, Guilment J (1991) Interactive self-modeling mixture analysis. Anal Chem 63:1425-1432

Wold S, Sjöström M, Eriksson L (2001) PLS-regression: a basic tool of chemometrics. Chemometr Intell Lab Syst 58:109-130 